\title{
Experimental evidence of two types of photorefractive effects occuring during pulsed or CW photoexposures of Bragg gratings within germanosilicate fibres
}

\author{
P. NIAY, P. BERNAGE, W.X. XIE, S. LEGOUBIN, M. DOUAY, J.F. BAYON*, T. GEORGES* and \\ M. MONERIE*
}

Laboratoire de Dynamique Moléculaire et Photonique, URA 779 du CNRS, Université des Sciences et Technologies de Lille, UFR de Physique, Bâtiment P5, 59655 Villeneuve d'Ascq cedex, France

* LAB/OCM/FOG, CNET-Lannion, Route de Trégastel, 22301 Lannion cedex, France

Photoinscriptions of Bragg gratings have been carried out within several germanosilicate fibres via UV side writing near $244 \mathrm{~nm}$. The gratings have been written by side exposure of the core of the fibre to a UV two beam interference pattern from a pulsed or a CW laser. Real time, high resolution measurements of the spectral transmission, of the F.W.H.M. bandwidth and of the resonance wavelengths of the Bragg gratings lead to noteworthy observations when long irradiation times are used.

Second order diffraction efficiency has been observed from Bragg gratings written within some fibres. Second order diffraction spectrum can be detected once the first order grating reflectivity is just beginning to saturate. The evolution of the first (and second) order spectral transmissions of the Bragg gratings, as a function of exposure time, looks very complex in some fibres. Indeed, long irradiation time leads to the formation of a first order (and of a second order) spectrum followed by its erasure and then by a new spectrum formation. The Bragg wavelengths of gratings increase with irradiation time up to the time when partial or complete erasure of the first order spectrum occurs. On the other hand, the Bragg wavelengths remain stationary or decrease in the course of the new formation of the first order spectrum. These evolutions as a function of irradiation time, are taken into account by using a phenomenological model which assumes that two photorefractive effects should be responsible for the grating growth. To check the validity of this assumption, bleaching and thermal annealing experiments have been performed using gratings written under various conditions in the fibres for which complex evolutions of spectra could be observed. One can note, that the stability of the experimental set-up needed for these experiments, is not as critical as when writing gratings by exposures of the fibre to a fringe pattern.

UV transverse fringeless irradiation of gratings first erases the first order spectrum. Afterwards, depending on the parameters of the irradiation used to 
write the grating, fringeless irradiation can build or not a new spectrum. Similarly, isochronal thermal annealing experiments have shown that first order spectrum reflectivities of gratings can increase dramatically after a rise of $525^{\circ} \mathrm{C}$ in the fibre temperature. The model is consistent with these observations, if one assumes that the thermal stabilities of the defect centres responsible for the two photorefractive effects are different. The major part of the results concerns experiments performed using a pulsed UV laser but preliminary observations from gratings written using CW UV commercial or home-made sources show that the features of the spectrum evolutions with irradiation time are similar whatever the nature of the source may be.

Aknowledgments: We wish to aknowledge coherent SA France for lending a CW UV source (F.R.E.D.) with which a part of the results was obtained. Dr B. POUMELLEC is aknowledged for fruiful discussions. The work is supported by a C.N.E.T. contract $(938 \mathrm{~B} 006)$ and a D.R.E.T. contract (92147). 